\title{
ENDOMETRIOSIS OF THE RECTUS ABDOMINIS MUSCLE: A RARE CAUSE OF EMERGENCY DEPARTMENT VISIT
}

\author{
Murat Seyit ${ }^{1}$, Yalcin Sonmez $^{2}$, Atakan Yilmaz $^{1}$, Mert Ozen $^{1}$ \\ ${ }^{1}$ Department of Emergency Medicine, Pamukkale University, Denizli, Turkey \\ ${ }^{2}$ Department of General Surgery, Dumlupınar University, Kütahya, Turkey
}

\section{CASE REPORT}

Doi: $10.33695 /$ rojes.v2i2.25

Accepted: 11.10 .2020

\section{Corresponding author: \\ Murat Seyit, E-mail:} muratseyit@yahoo.com

\begin{abstract}
Endometriosis affects approximately $10 \%$ of women of reproductive age. Although the most common affected sites are ovaries, uterosacral ligament, rectovaginal septum, and pelvic peritoneum, endometriosis can be located in any organ. Endometriosis of the rectus abdominis muscle independent from the cesarean section incision is extremely rare. A 31-years-old female was admitted to the emergency department complaining of periodical abdominal pain and distension. Ultrasonography and computed tomography revealed a heterogeneous mass in the rectus abdominis muscle. Surgical excision was performed. The histopathologic examination showed endometrial tissue with glandular and stromal elements within the muscularis propria. Endometriosis should be kept in mind in the differential diagnosis of atypical pain and mass of the abdominal wall in emergency settings.
\end{abstract}

Keywords: Emergency service, endometriosis, pain, rectus abdominis muscle

\section{Introduction}

The presence and growth of endometrial tissue outside the uterus is called endometriosis. It affects women of reproductive age and - with a $10 \%$ prevalence rate - is not an unusual gynecological disease. Although the most common affected sites for endometriosis are ovaries, uterosacral ligament, rectovaginal septum, and pelvic peritoneum, it can be located in any organ including stomach, liver, and bladder. This ectopic functional endometrial tissue is often associated with inflammation, severe and chronic pain, and infertility [1]. In approximately $6 \%$ of cases, endometriosis is located outside the genital organs and pelvis [2].
Pain is the most common cause of emergency visit and one of the most prevalent symptoms of endometriosis. On clinical presentation, endometriosis may simulate a variety of inflammatory, infectious and neoplastic conditions. Misdiagnosis is not uncommon, especially when it is present outside the pelvis. We report an atypical presentation of a 31-years-old female to the emergency department, with endometriosis of the rectus abdominis muscle. Interestingly, in this case, the endometriosis tissue is independent of the cesarean scar.

\section{Case Presentation}

A 31-years-old nurse was admitted to the emergency department due to abdominal pain and distension. She had a history of acute 
lymphoblastic leukemia and breast malignancy. (both in remission), and a cesarean section 3 years ago (gravida 1, para 1, abortus 0). She complained of abdominal pain (lasting a few days during the menstrual period), local distention (for about two years), and a palpable mass $5 \mathrm{~cm}$ above the cesarean section incision that was not related to the cesarean section.

On physical examination, findings were normal. Abdominal ultrasonography (USG) revealed a 30x10 $\mathrm{mm}$ heterogeneous hypoechoic mass with irregular contours in the right rectus abdominis muscle of the suprapubic region (Figure 1). Valsalva maneuver did not yield any change in the mass. Abdominal computed tomography (CT) showed a heterogeneous mass in the right rectus abdominis muscle which was consistent with endometrioma (Figure 2).

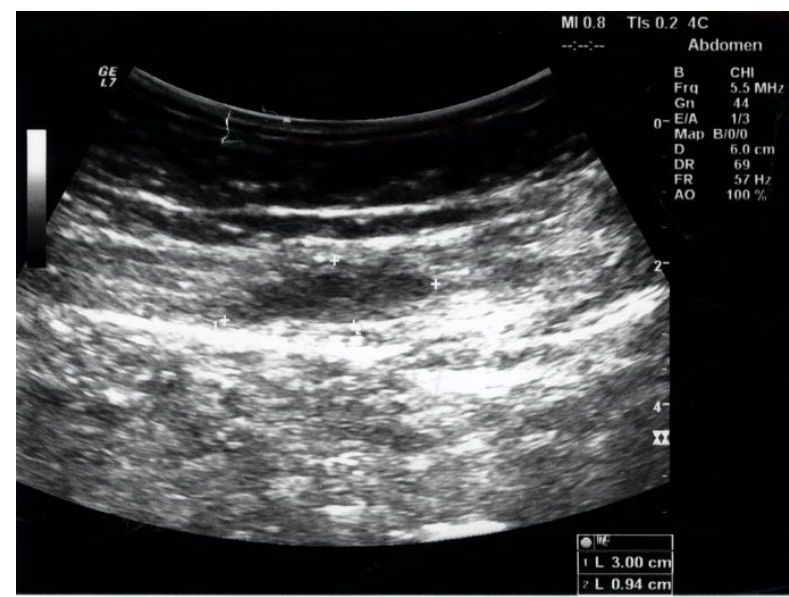

Figure 1 - Abdominal ultrasonography image of the $30 \times 10 \mathrm{~mm}$ heterogeneous hypoechoic mass with irregular contours in the right rectus abdominis muscle

Considering history and imaging modalities, the presumed diagnosis of endometrioma was made. We performed surgical excision under general anesthesia, through a median incision below the umbilicus. The mass adhered to the rectus abdominis muscle and peritoneum; limited peritoneal excision was also performed. The histopathologic examination of the mass $(4 \mathrm{x}$
$3.5 \times 2.5 \mathrm{~cm}$ in diameter) showed cellular endometriosis surrounded by fibromuscular tissue and collagen fibers (Figure 3). An oral contraceptive preparation was prescribed to the patient postoperatively. No relapse was observed during the follow-up of 6 months.

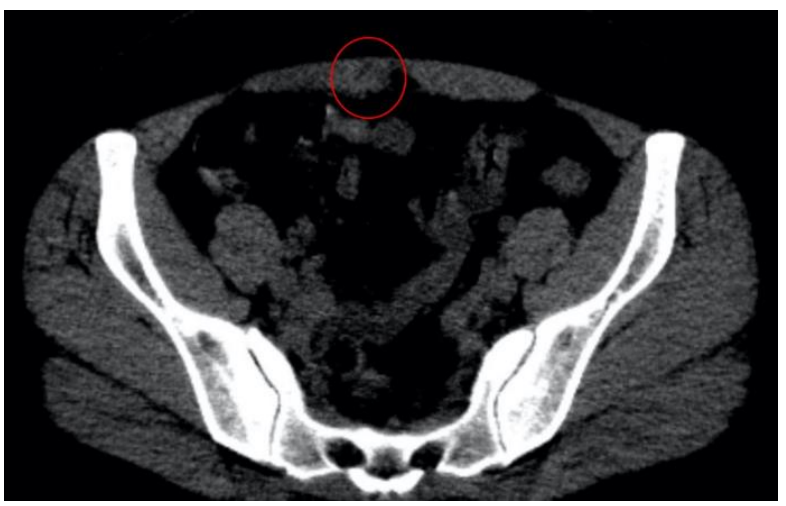

Figure 2 - Abdominal computed tomography section shows heterogeneous mass in the right rectus abdominis muscle.

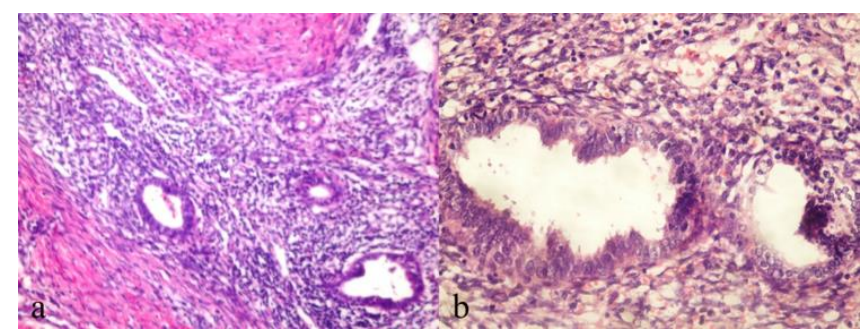

Figure 3

a: Endometrial tissues with glandular and stromal elements are observed within the muscularis propria (Hematoxylin\&eosin, original magnification $\mathbf{x 1 0 0}$ ).

3b: Glands are formed by single layer endometrial cells without metaplastic or atypical changes (Hematoxylin\&eosin, original magnification $x 100$ ).

\section{Discussion}

Abdominal wall involvement by functional endometrial tissue over Pfannenstiel incision was first described by Aimakhu in 1975 [3]. Endometriosis of the anterior abdomen over cesarean incision constitutes $1 \%$ of all patients with extra-genital endometriosis [4]. However, isolated rectus abdominis muscle involvement by 
endometriosis independent from the incision site is rare. Although both conditions have clinical and symptomatic similarities, the underlying mechanism is entirely different. Many theories exist for the development of endometriosis including metaplasia, induction and transplantation theories. Although the exact pathogenetic mechanism of rectus abdominis endometriosis is not known, the most possible reason is the spread of endometrial cells through cesarean section incision or vascular migration. In the present case, the functional endometrial tissue was 5 $\mathrm{cm}$ above the cesarean incision, therefore it is presumed to be secondary to the vascular spread.

The pathological processes associated with pelvic endometriosis are inflammation and fibrosis of the peritoneum and the formation of adhesions. Pelvic pain and infertility are the main symptoms that affect women's quality of life. Extragenital endometriosis may present with atypical pain related to the menstrual cycle due to continued growth of endometriosis tissue and its estrogen responsibility. Our patient had a history of abdominal pain (for a few days during the menstruation period) and a midline mass (for about two years). Not only gynecologists but also physicians in emergency settings should be familiar with the symptoms of extragenital endometriosis, because most of the patients with pain refer to the emergency department.

The first diagnostic tool for the diagnosis is USG, but CT and dynamic magnetic resonance imaging are other useful diagnostic methods. Fine needle aspiration biopsy is a valuable invasive approach to confirm the diagnosis. The diagnosis is confirmed by the histopathologic examination, which shows endometrial glands and stroma. In this case, the diagnosis was made based on the history, USG, CT and histopathologic sections. Careful history taking and physical examination are critically important. Finally, endometriosis should be kept in mind in the differential diagnosis of atypical pain associated with the menstrual cycle in women of reproductive age.

Papavramidis et al. [5] excised a $5 \times 4 \mathrm{~cm}$ mass in the abdominal region of a 28 -year-old woman, that had no previous surgical history. The histopathological evaluation of the excision material confirmed endometriosis. What makes our case different is that endometriosis is in the rectus muscle.

Bozkurt et al. [6] reported a 25-years-old female who was diagnosed with intramuscular abdominal wall endometriosis through history, physical exam, USG and magnetic resonance imaging. The patient was treated by USGguided intralesional 95\% ethanol injection. The authors reported no recurrence during the 9-months follow up period. Coccia and colleagues [7] reported USG-guided removal of rectus abdominis endometriosis for the first time in literature: rectus abdominis endometriosis was treated by surgical excision and no recurrence was observed during the 6months follow up period.

\section{Conclusion}

Although endometriosis is known to be a benign disease, malignant characteristics such as invasion, migration, metastasis and recurrence may be observed. Rectus abdominis muscle involvement by endometriosis is rare. We should keep it in mind in the differential diagnosis of atypical pain and mass of the abdominal wall. It is interesting to note the appearance of endometrial tissue independent of the cesarean scar.

\section{References}

[1] M. Hickey, K. Ballard, and C. Farquhar, 'Endometriosis', BMJ, vol. 348, p. g1752, Mar. 2014.

[2] H. Karaman, F. Bulut, and A. Özaşlamac1, 'Endometriosis externa within the rectus abdominis muscle', Turk. J. 
SurgeryUlusal Cerrahi Derg., vol. 30, no. 3, pp. 165-168, Sep. 2014.

[3] V. E. Aimakhu, 'Anterior abdominal wall endometriosis complicating a uteroabdominal sinus following classical cesarean section', Int. Surg., vol. 60, no. 2, pp. 103-104, Feb. 1975.

[4] K. E. Koger, C. H. Shatney, K. Hodge, and J. H. McClenathan, 'Surgical scar endometrioma', Surg. Gynecol. Obstet., vol. 177, no. 3, pp. 243-246, Sep. 1993.

[5] T. S. Papavramidis et al., 'Spontaneous abdominal wall endometriosis: a case report',
Acta Chir. Belg., vol. 109, no. 6, pp. 778-781, Dec. 2009.

[6] M. Bozkurt, A. S. Çil, and D. K. Bozkurt, 'Intramuscular Abdominal Wall Endometriosis Treated by Ultrasound-Guided Ethanol Injection', Clin. Med. Res., vol. 12, no. 3-4, pp. 160-165, Dec. 2014.

[7] M. E. Coccia, F. Rizzello, S. Nannini, M. Cozzolino, T. Capezzuoli, and F. Castiglione, 'Ultrasound-guided excision of rectus abdominis muscle endometriosis', J. Obstet. Gynaecol. Res., vol. 41, no. 1, pp. 149-152, Jan. 2015. 